\title{
Is orthodontic treatment a risk factor for temporomandibular disorders?
}

\author{
Tomas Magnusson ${ }^{1}$, Antonio Sérgio Guimarães ${ }^{2}$
}

Introduction: The possibility that orthodontic treatment in childhood might be a risk factor for the development of temporomandibular disorders (TMD) later in life has been an issue of great controversy in dental literature.

Objective: To determine a possible negative or positive correlation between orthodontic treatment and TMD by presenting the results and conclusions from a number of key-papers dealing with this subject.

Results and Conclusion: According to current knowledge, there is no scientific evidence to support that orthodontic treatment is a risk factor for the development of TMD. On the other hand, there is some evidence to support that a proper orthodontic treatment performed in childhood might have a positive effect upon the functional status of the masticatory system later in life.

Keywords: Temporomandibular disorder. Occlusion. Orthodontic treatment.

${ }^{1} \mathrm{PhD}$ and Professor, Department of Stomatognathic Physiology, The Institute for Posgraduate Dental Education and School of Health Sciences, Jönköping University, Jönköping, Sweden.

${ }^{2} \mathrm{PhD}$ and Professor. Director of the Temporomandibular Disorder and Orofacial Pain Clinic of the Hospital São Paulo, FUNIFESP, São Paulo, Brazil. Director of the Tempormandibular Disorder and Orofacial Pain Clinic of the Hospital São João, University of Porto City, Porto City, Portugal. Course Director of the MsC Program in TMD and Orofacial Pain, São Leopoldo Mandic University, Campinas, Brazil.
How to cite this article: Magnusson T, Guimarães AS. Is orthodontic treatment a risk factor for temporomandibular disorders? Dental Press J Orthod. 2012 MarApr;17(2):97-103.

Submitted: September 15, 2008 - Revised and accepted: March 9, 2009

» The authors report no commercial, proprietary, or financial interest in the products or companies described in this article.

Contact address: Antônio Sérgio Guimarães

Rua França Pinto, 1085/61, Vila Mariana - São Paulo / SP - CEP: 04.016-034 - Brazil E-mail:asgatm@gmail.com 


\section{INTRODUCTION}

The opinion that orthodontic treatment can cause temporomandibular disorders (TMD) is widespread among general dental practitioners. Likewise, this has been frequently mentioned in literature, and some studies done by Franks ${ }^{1}$ are often used to support this statement. In this paper, it was reported that $11 \%$ out of 751 individuals with joint dysfunction had a previous experience of orthodontic treatment, while only $2 \%$ of 326 individuals without dysfunction had received orthodontic treatment in childhood. Several authors have interpreted these results as evidence that orthodontic treatment is a risk factor for TMD later in life. This would be an incorrect assumption since that author wrote on the very same paper that "the great variation on the type and period of the orthodontic treatments performed prevented a formal analysis of these data".

The question about the possible correlation becomes even more confusing since the opposite opinion, that orthodontic treatment can cure and/or even prevent TMD, has also been claimed. It has been stated, for instance, that "if we have the concept of building an occlusion to fit the mandible mechanism, the TMJ pain-dysfunction syndrome can be virtually eliminated in the post-orthodontic patient". ${ }^{2}$

During the years a great number of papers have focused on the possible negative or positive relations between orthodontic treatment and TMD. A search on PubMed using the following keywords "orthodontics" and "TMD" resulted in almost 400 papers. Out of these, the ones dealing with different aspects of orthodontic treatment, morphological malocclusions and TMD were chosen; also only papers in English were used. The papers selected were denying a possible correlation between orthodontic treatment and TMD. However, it should be stressed that due to the large number of papers in this area, it was not possible to review completely the literature in these topics.

\section{LITERATURE REVIEW}

\section{Retrospective investigations}

Several retrospective papers have compared individuals that underwent orthodontic treatment with untreated individuals in respect of signs and symptoms of TMD. In an early investigation, ${ }^{3}$
23 previously treated individuals were examined for signs and symptoms of TMD 10 years after treatment. It was found that $31 \%$ of the patients exhibited mild clinical signs of TMD and 4\% showed severe signs. Twenty-seven percent of the patients also reported mild subjective symptoms of TMD. These data was compared to retrospective data from epidemiological investigations, and the authors concluded that there might be only a small increase on occurrence of TMD in patients subjected to orthodontic treatment. This paper has, on the other hand, some major drawbacks. The sample size was very small and no matched control group was included.

In a later study, 51 individuals ageing 19-year-old who had received previous orthodontic treatment, on average 5 years earlier, were compared for TMD signs and symptoms with 47 untreated individuals ageing 19-year-old also. ${ }^{4}$ Out of those who had previous treatment, $70.6 \%$ showed mild or moderate signs of dysfunction against $53.2 \%$ on the controls. This difference, however, was not significant. When asked for subjective symptoms, $70.6 \%$ of the treated patients and $91.5 \%$ of the controls reported mild symptoms of TMD such as TMJ sounds and feeling of fatigue and/or stiffness in masticatory muscles. This difference was significant, but since only mild symptoms were reported, the authors concluded that there were no substantial differences as to signs and symptoms of TMD between the two groups. These findings are in agreement to other studies ${ }^{5}$ with similar design, and the overall opinion is that there seems to be very few reasons to fear an increase in TMD signs and symptoms on patients subjected to orthodontic treatment. On the other hand, some of these retrospective studies suggest that the functional status of treated patients was more harmonious than in controls, and thus, no relation exists between TMD and a well-planned orthodontic treatment.

\section{Prospective investigations}

There is always a risk of being misguided when interpreting data from retrospective studies. Moreover, such studies do not supply information about the functional status before orthodontic treatment. During the 90's, the first prospective investigations focusing on orthodontic treatment and TMD were published. 
Among those, there are a series of papers published by Olsson and Lindqvist. ${ }^{6}$ They compared signs and symptoms of TMD of 245 individuals in need of orthodontic treatment with 245 matched controls. They found that $52.4 \%$ of the patients who were to about to start orthodontic treatment showed moderate or severe clinical signs of TMD, while $16.7 \%$ experienced only subjective symptoms. The values for the controls were $29.4 \%$ and $4.9 \%$, respectively. Since these differences were significant, the authors concluded that the patients in need of orthodontic treatment appear to be at greater risk of TMD, when compared to controls. The 245 individuals who were in need of orthodontic treatment were followed longitudinally ${ }^{7}$ and were treated with fixed orthodontic appliances. The final examination was made on an average of 4 years after the beginning of treatment, when the patients had been out of retention for 3-5 months, and when the occlusion had been stable for at least 6 months. Final data was collected from a total of 210 patients out of the original 245. The data showing moderate or severe signs of TMD had decreased from $45.2 \%$ to $20.0 \%$, and the reports of subjective symptoms had decreased from 16.7 to $6.7 \%$. That decrease of both signs and symptoms was significant, and the results were in fairly good agreement with those originally found among the control individuals. The authors stated that "if there is a smaller prevalence of symptoms of TMD, we believe that some credit has to been given to the orthodontic treatment", and they also concluded that such treatment can prevent further development of TMD to some extent, or even cure it.

\section{Longitudinal epidemiological studies}

Some longitudinal epidemiological investigations have also focused on possible correlations between orthodontic treatment and TMD. In one study, a total of 402 children in 3 age groups, originally 7-, 11- and 15-year-olds, were followed for 20 years. ${ }^{8,9}$ During this time period, approximately onethird of the total sample received some kind of orthodontic treatment. Sixty percent of the treatments were performed by specialists and $40 \%$ by general practitioners. At a 10 year follow-up, ${ }^{8}$ it was shown that subjective symptom of TMD had increased in all 3 age-groups, but such symptoms were more pronounced in untreated individuals. The signs reported were the same while a decrease was perceived in those who had undergone treatment. At the 20 year follow-up, ${ }^{9}$ however, these differences were levelled out, and no difference could be found in respect of signs and symptoms of TMD between treated and untreated individuals. These findings were in agreement with another longitudinal study ${ }^{10}$ where a group of patients treated by specialists were compared with a matched control group, with ages from 15 to 18 years old, after treatment.

\section{Extraction versus non-extraction}

The possible consequences of orthodontic treatment with or without extractions on TMD have also been discussed in literature, and the results were not conclusive.

In one study, ${ }^{11}$ individuals with Class II malocclusions developed TMD after treatment with extraction of 2 upper premolars and retroclination of the incisors with removable appliances. The authors suggested that this was due to a greater than average overbite that resulted from treatment.

In a study by Janson and Hasund, ${ }^{12}$ the functional consequences of different orthodontic interventions were investigated. A group of 90 individuals with Class II Division 1 malocclusion were assigned to one of 3 groups: No treatment, orthodontic treatment including extraction of 4 premolars and non-extraction treatment. The results were evaluated after 5 years and it was found that those treated with non-extraction therapy had less signs and symptoms of TMD compared to the other 2 groups, and those treated with extractions showed less signs and symptoms of TMD when compared to controls. They concluded that a non-extraction approach should be aimed for whenever possible.

On the other hand, no difference could be found between the groups in respect of TMD, in a more recent publication ${ }^{13}$ comparing non-extraction treatment to treatment with 2 or 4 extractions.

In a more elaborate longitudinal study ${ }^{14} 172$ treated individuals were followed for 20 years in order to evaluate the effects of extraction or non-extraction on TMD. No differences were found between the groups, and the conclusion was that neither treatment caused differences in signs and symptoms of TMD. 


\section{Orthodontic treatment performed by orthodontists versus general practitioners}

It is fairly common in Sweden for general practitioners (GPs) to perform orthodontic treatment under the supervision of orthodontists, which is mostly done with removable appliances. The possible difference in functional status after orthodontic treatment performed by orthodontists or GPs has been investigated.

One study ${ }^{15}$ showed data from a Swedish population of 1554 19-year old individuals. The more complex cases $(60 \%)$, with or without extractions, were treated with fixed appliances by orthodontists, while less complex cases (40\%) were treated by general practitioners under the supervision. These treatments consisted mostly of removable appliances. After completion of the treatments, the prevalence of both signs and symptoms of TMD were equal between the groups, and their signs and symptoms were also comparable to matched untreated controls.

\section{TMD during orthodontic treatment}

The prevalence of TMD signs and symptoms, including tension-type headache, has also been studied longitudinally before, during and after orthodontic treatment. ${ }^{16,17}$ The conclusion from these studies is that there is a significant decrease of signs and symptoms of TMD and of the headache during the active phase of treatment, but they tend to increase to pre-treatment levels after treatment is finished.

\section{Functional and morphological malocclusion and their possible relation on the etiology of TMD}

The effects of occlusal factors on the function of the masticatory system have been a controversial topic over the years. The several existing epidemiological investigations have not been able to demonstrate any strong correlations between different functional malocclusions/occlusal interferences and TMD. ${ }^{18}$ However, some weak but statistically significant associations have been found between TMDs and a large sagittal distance between retruded contact position (RCP) and intercuspal contact position (ICP) ${ }^{19,20}$ as well as between TMDs and a lateral shift between RCP and ICP., ${ }^{918}$

From an orthodontic point of view, the possibility of an effect of the morphological malocclusions in the development of TMD is of great interest, and some specific morphological malocclusions have been found to be associated with TMD variables. Mohlin and Kopp ${ }^{21}$ found that anterior open bites and posterior crossbites were more common in TMD patients compared to healthy controls. Studies by Tanne et al, ${ }^{22}$ as well as Pullinger et al, ${ }^{23}$ also agree with the finding that these 2 malocclusions, as well as a deep bite, are significantly associated with the occurrence of TMD.

Longitudinal studies following the same individuals for 20 years have found that individuals with mandibular deficiency have a much higher risk for tooth wear (odds ratio 7.3) compared to individuals with normal occlusion, ${ }^{24}$ and that individuals with posterior crossbites developed two times more TMJ-related problems when compared to normal occlusion (57\% and $21 \%$, respectively). ${ }^{25}$ Finally, they found that individuals with a deep bite had a much greater risk for developing clinical signs of dysfunction (odds ratio: 12.5) compared to those with no malocclusion. ${ }^{26}$

\section{DISCUSSION}

From early retrospective investigations of previously treated patients, ${ }^{3,4,5}$ it was concluded that patients that underwent orthodontic treatment do not exhibit more signs or symptoms of TMD compared to untreated individuals. However, from the findings in later prospective studies ${ }^{4}$ it can be speculated that the similarities regarding TMD in patients and controls found in the early retrospective studies was in fact the consequence of an improvement of TMD among those patients treated, making them, in respect of TMD, equal to individuals subjected to orthodontic treatment.

Prospective and longitudinal studies ${ }^{6-10}$ lend further support to the opinion that individuals who have undergone treatment do not have a higher risk of developing TMD later in life. An alternative view of this issue is that individuals who needed and received orthodontic treatment as children had the same risk for developing TMD, when adults, as untreated individuals.

The literature is inconclusive regarding functional advantages or disadvantage in extractions or no extractions associated with orthodontic therapy. ${ }^{11-14}$ The main impression is that a well planned treatment, irrespective if extraction was part of the 
treatment or not, does not have any negative influence on the function of the masticatory system.

An interesting conclusion from literature is that no higher risk exists for development of signs and symptoms of TMD if a GP is performing the orthodontic treatment (in specific cases) compared with orthodontists, or if their patients are compared to untreated controls. ${ }^{15}$

Another finding, which was unexpected, is that both signs and symptoms of TMD decrease during the orthodontic treatment, but tend to return to pre-treatment levels after treatment. ${ }^{16,17}$ The reasons for this might be numerous, but the most likely explanation is that the teeth which are being moved become sensitive to occlusal loading, what causes a decrease of the oral parafunctions during the time of active treatment.

Correlations between both functional and morphological malocclusions and signs and symptoms of TMD are, with few exceptions, weak or non-existent., ${ }^{9-20-23}$ There is, however, some indications that a large sagittal distance between RCP and ICP, as well as a lateral shift between those positions, might add to a risk for developing TMD., ${ }^{918-21}$ Thus, these functional malocclusions should, when possible, be treated orthodontically.

Morphologically, the anterior open bite, the deep bite, the mandibular deficiency, and the crossbite involve an increased risk for different TMDs to occur..$^{20,22-26}$ It should be emphasized that, with the exception for posterior crossbite, the increased risk is smaller. Thus, an orthodontic treatment should not be accomplished under the argument that it will prevent TMD problems later in life.

Even though the risk is small for the orthodontic treatment to cause TMDs, recording any signs and/ or symptoms of TMD before the start of the orthodontic treatment is recommended. One of the reasons for that is to avoid that a patient should claim that symptoms have developed as a consequence of the treatment when they were actually present at start of treatment. Symptoms that should be recorded are frequent headache, pain in the face or mandibles, painful mandible movements, and TMJ sounds.

The procedures on how to perform a functional examination of the masticatory system have been described in TMD textbooks. ${ }^{27,28}$ The examination should at least include measurement of maximal mandible opening; registration of palpatory pain on the TMJs, on the superficial part of the masseter muscle, and on the insertion of the temporalis muscle; recordings of TMJ sounds; and painful mandible movements.

It is important to underline that even when there is a clear presence of TMD signs and symptoms, this is not an absolute contraindication to orthodontic treatment because as stated above, the mobility of the teeth during treatment will cause parafunctional activities such as clenching and/or grinding of teeth to diminish or cease.

It is well known from the evidence, in many cross-sectional and longitudinal epidemiological investigations concerning TMD, that the prevalence of both signs and symptoms of TMD increase from childhood through adolescence and into young adulthood, where it seems to level out and decrease. ${ }^{29}$ The incidence, or in other words, the number of new TMD cases/year has been found to be approximately $1 \% .^{30}$

This increase in TMD signs and symptoms coincide with the age in which the vast majority of the patients undergo orthodontic treatment. Thus, it can be expected that a number of individuals receiving treatment will develop TMD, either during or shortly after the treatment, due to chance. Moreover, even in specific cases it is not possible, most of the time, to judge if a TMD was caused by an orthodontic treatment or not.

If painful TMD symptoms and/or functional impairment should develop during the active treatment phase, it is recommended to modify the treatment, to reduce forces or even to interrupt it for some time. The occlusion should also be checked for large interferences, which, if present, should be eliminated with selective occlusal grinding.

If TMD symptoms should start during or shortly after the retention phase, it is recommended to re-evaluate both the retention appliance and the final occlusion.

It should be strongly emphasized that there is a great possibility that any signs and symptoms of TMD starting during orthodontic treatment might be happening due to a time coincidence rather than because of the therapy. 


\section{CONCLUSION}

In agreement with several and previous systematic reviews, ${ }^{31-35}$ this paper clearly shows that orthodontics, either with or without extractions, does not cause TMD.
Moreover, there is some evidence to support that a proper orthodontic treatment performed in childhood might have a positive effect upon the functional status of the masticatory system later in life. 


\section{REFERENCES}

1. Franks AS. The dental health of patients presenting with temporomandibular joint dysfunction. Br J Oral Surg. 1967;5(2):157-66

2. Aubrey RB. Occlusal objectives in orthodontic treatment. Am J Orthod. 1978;74(2):162-75.

3. Larsson E, Ronnerman A. Mandibular dysfunction symptoms in orthodontically treated patients ten years after the completion of treatment. Eur J Orthod. 1981;3(2):89-94.

4. Dahl BL, Krogstad BS, Ogaard B, Eckersberg T. Signs and symptoms of craniomandibular disorders in two groups of 19-year-old individuals, one treated orthodontically and the other not. Acta Odontol Scand. 1988;46(2):89-93.

5. Kess K, Bakopulos K, Witt E. TMJ function with and without orthodontic treatment. Eur J Orthod. 1991;13(3):192-6.

6. Olsson M, Lindqvist B. Mandibular function before orthodontic treatment. Eur J Orthod. 1992;14(1):61-8

7. Olsson M, Lindqvist B. Mandibular function before and after orthodontic treatment. Eur J Orthod. 1995;17(3):205-14

8. Egermark I, Thilander B. Craniomandibular disorders with special reference to orthodontic treatment: an evaluation from childhood to adulthood. Am J Orthod Dentofacial Orthop. 1992;101(1):28-34.

9. Egermark I, Magnusson T, Carlsson GE. A 20-year follow-up of signs and symptoms of temporomandibular disorders and malocclusions in subjects with and without orthodontic treatment in childhood. Angle Orthod. 2003;73(2):109-15.

10. Egermark I, Carlsson GE, Magnusson T. A prospective long-term study of signs and symptoms of temporomandibular disorders in patients who received orthodontic treatment in childhood. Angle Orthod. 2005;75(4):645-50.

11. Berry DC, Watkinson AC. Mandibular dysfunction and incisor relationship. A theoretical explanation for the clicking joint. Br Dent J. 1978;144(3):74-7.

12. Janson M, Hasund A. Functional problems in orthodontic patients out of retention Eur J Orthod. 1981;3(3):173-9.

13. Kremenak CR, Kinser DD, Harman HA, Menard CC, Jakobsen JR. Orthodontic risk factors for temporomandibular disorders (TMD). I: Premolar extractions. Am J Orthod Dentofacial Orthop. 1992;101:13-20.

14. Dibbets JM, van der Weele LT. Long-term effect of orthodontic treatment including extraction, on signs and symptoms attributed to CMD. Eur J Orthod 1992;14(1):16-20.

15. Lagerstrom L, Egermark I, Carlsson GE. Signs and symptoms of temporomandibular disorders in 19-year-old individuals who have undergone orthodontic treatment. Swed Dent J. 1998;22(5-6):177-86.

16. Egermark I, Ronnerman A. Temporomandibular disorders in the active phase of orthodontic treatment. J Oral Rehabil. 1995;22:613-8.

17. Henrikson T, Nilner M, Kurol J. Symptoms and signs of temporomandibular disorders before, during and after orthodontic treatment. Swed Dent J. 1999;23:193-207.
18. Seligman DA, Pullinger AG. The role of functional occlusal relationships in temporomandibular disorders: a review. J Craniomandib Disord. 1991;5(4):265-79.

19. Magnusson T, Carlsson GE, Egermark-Eriksson I. An evaluation of the need and demand for treatment of craniomandibular disorders in a young Swedish population. J Craniomandib Disord. 1991;5:57-63.

20. Pullinger AG, Seligman DA, Gornbein JA. A multiple logistic regression analysis of the risk and relative odds of temporomandibular disorders as a function of common occlusal features. J Dent Res. 1993;72(6):968-79.

21. Mohlin B, Kopp S. A clinical study on the relationship between malocclusions, occlusal interferences and mandibular pain and dysfunction. Swed Dent J. 1978;2:105-12.

22. Tanne K, Tanaka E, Sakuda M. Association between malocclusion and temporomandibular disorders in orthodontic patients before treatment. J Orofac Pain. 1993;7(2):156-62.

23. Pullinger AG, Seligman DA. Quantification and validation of predictive values of occlusal variables in temporomandibular disorders using a multifactorial analysis. J Prosthet Dent. 2000;83(1):66-75.

24. Carlsson GE, Egermark I, Magnusson T. Predictors of bruxism, other ora parafunctions, and tooth wear over a 20-year follow-up period. J Orofac Pain. 2003;17(1):50-7.

25. Magnusson T, Egermark I, Carlsson GE. A prospective investigation over two decades on signs and symptoms of temporomandibular disorders and associated variables. A final summary. Acta Odontol Scand. 2005;63(2):99-109.

26. Carlsson GE, Egermark I, Magnusson T. Predictors of signs and symptoms of temporomandibular disorders: a 20-year follow-up study from childhood to adulthood. Acta Odontol Scand. 2002;60:180-5.

27. Carlsson GE, Magnusson T. Management of temporomandibular disorders in General Dental Practice. Chicago: Quintessence, 1999.

28. Carlsson GE, Magnusson T, Guimarães AS. Tratamento das disfunções temporomandibulares na clínica odontológica. São Paulo: Quintessence; 2006

29. Magnusson T, Egermark I, Carlsson GE. A longitudinal epidemiologic study of signs and symptoms of temporomandibular disorders from 15 to 35 years of age. J Orofac Pain. 2000;14:310-9.

30. Magnusson T, Egermark I, Carlsson GE. Treatment received, treatment demand and treatment need for temporomandibular disorders in 35-year-old subjects. Cranio. 2002;20(1):11-7.

31. McNamara JA Jr, Seligman DA, Okeson JP. Occlusion, orthodontic treatment, and temporomandibular disorders: a review. J Orofac Pain. 1995;9:73-90.

32. McNamara JA, Turp JC. Orthodontic treatment and temporomandibula disorders: Is there a relationship? Part 1. Clinical studies. J Orofac Orthop. 1997:58(2):74-89.

33. Kim MR, Graber TM, Viana MA. Orthodontics and temporomandibular disorders: A meta-analysis. Am J Orthod Dentofacial Orthop. 2002;121(5):438-46.

34. Henrikson T, Nilner M. Temporomandibular disorders, occlusion and orthodontic treatment. J Orthod. 2003 Jun;30(2):129-37; discussion 127.

35. Mohlin B, Axelsson S, Paulin G, Pietila T, Bondemark L, Brattstrom V, et al. TMD in relation to malocclusion and orthodontic treatment. Angle Orthod. 2007;77(3):542-8. 